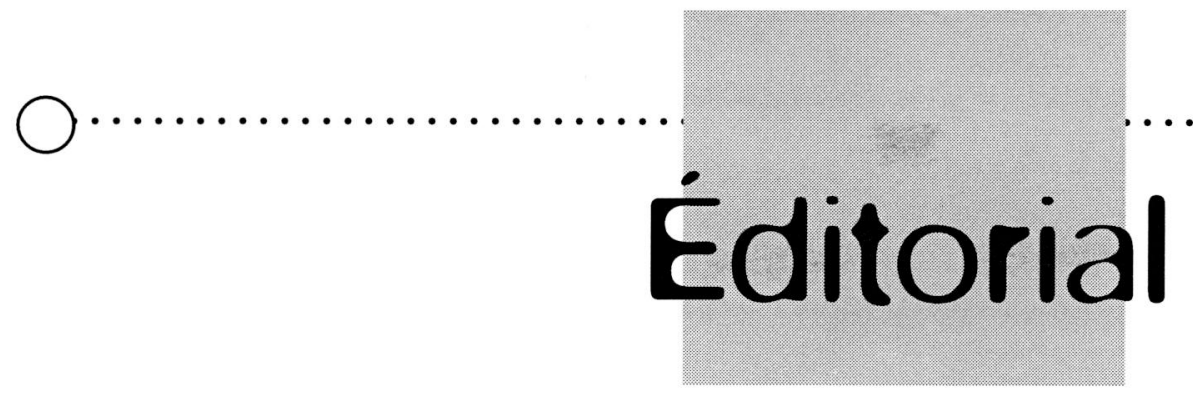

\title{
Vérité psychique et vérité judiciaire
}

*Psychiatre des Hôpitaux, Centre Psychiatrique du Bois de Bondy,

13-15, voie

Promenade, 93147 Bondy Cedex, France.

\author{
Daniel ZAGURY*
}

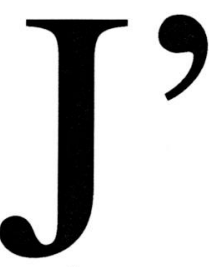

ai lu dans les comptes rendus du procès de l'affaire d'Outreau que les experts avaient estimé les enfants crédibles. C'est une information que l'on donne parfois - non sans ironie - lorsque des accusations semblent s'effondrer. Il me paraît nécessaire d'apporter quelques précisions et réflexions, car trop de malentendus pèsent sur la notion de crédibilité.

J'ai été expert dans quelques affaires surmédiatisées au moment de leur émergence publique qui se sont achevées quelques mois ou années plus tard par un non-lieu prononcé dans la plus stricte intimité : entre-temps, la prison, les vies brisées et l'ombre tenace d'une suspicion qu'aucune décision de justice ne pourra effacer.

Expert dans une affaire du sud-ouest qui a défrayé la chronique, je n'en évoquerai pas ici le cas mais seulement l'écho médiatique : j'ai cru assister pendant quelques jours à l'irruption d'un véritable délire collectif, quand un tueur en série a été érigé en Maître de la Ville, organisant des bacchanales sadiques avec les personnages les plus haut placés, en bénéficiant des plus sûres protections. Fritz Lang en aurait fait un chef d'œuvre. Là encore, la presse a souligné dans l'après-coup que les experts ayant examiné les victimes présumées avaient conclu à leur crédibilité au sens médico-légal, comme si cela authentifiait leurs déclarations.

Mais surtout, comme tous mes collègues, je suis régulièrement nommé pour des affaires dans lesquelles, parole de l'un contre parole de l'autre, l'expert est placé en arbitre suprême, bien à tort. À la lecture de dossiers, j'ai parfois le sentiment qu'une caricature en a remplacé une autre : à l'expert psychiatre si décrié pour commettre « un deuxième viol », par ses questions intrusives ou son incrédulité dévastatrice, a fait suite l'expert psychiatre ou psychologue angélique, pour qui le doute même est inconcevable. Il y a un enjeu crucial autour de ces questions, car nous risquons de jeter le bébé avec l'eau du bain des effets pervers du progrès : dans notre pays, des vagues successives ont permis en quelques décennies de faire sortir du silence le viol, l'inceste puis la pédophilie. Dernière en date des levées de refoulement collectif, l'apparition sous les regards horrifiés des abus sexuels commis par des mères sur leurs enfants, comme s'il n'était pas naîf de croire que les femmes étaient exclues de toute violence sexuelle agie. Un tel déni ôterait d'ailleurs toute existence propre à la sexualité féminine. Canguilhem [1], philosophe et médecin, a bien montré que nous ne connaissons la norme qu'à partir de l'expression de ses transgressions.

Autrement dit, on peut soutenir sans absurdité que s'il n'y avait pas quelques femmes pour commettre des horreurs sexuelles - elles aussi - c'est que toutes les femmes seraient dépourvues de sexualité. Regarder la vérité en face est toujours un progrès. C'est ce qui a permis un meilleur accueil des victimes présumées, une meilleure connaissance des diverses formes de délinquance sexuelle, une plus grande facilité à les dénoncer, un espoir de mieux les combattre. Mais gare au retour de balancier si l'on se met à tout confondre, et notamment vérité psychique et vérité judiciaire. La question ne date pourtant pas d'hier. En 1906, Freud fait une conférence devant un parterre de juristes sur l'application possible de la psychanalyse à l'établissement des faits en matière judiciaire [2]. Il se livre à une analogie entre la démarche du juge d'instruction et celle du psychanalyste, entre le secret du criminel et celui de l'hystérique. Mais il met en garde son auditoire contre une limite de taille à cette méthode : le sentiment de culpabilité du névrosé susceptible d'égarer le juge. Il donne alors libre cours à son 
scepticisme, exécutant en quelques phrases une méthode dénuée de portée pratique dans l'exercice de la justice, compte tenu du risque d'erreur.

Plus près de nous, l'exemple américain témoigne $a$ contrario de ce que l'inventeur de la psychanalyse avait été bien avisé de refuser tout amalgame entre vérité de l'inconscient et vérité des faits. Dans les années 1980, des psychothérapeutes et des experts, réunis dans le « mouvement de la mémoire retrouvée », ont pu soutenir devant les tribunaux que tous les souvenirs d'abus sexuels émergents étaient les rejetons de la réalité historique. Telle jeune femme qui avait rêvé que son père la violait avec un crucifix ne pouvait qu'avoir été l'objet de manœuvres incestueuses. Quelques erreurs judiciaires retentissantes plus tard, un autre lobby, la « fondation du faux syndrome de la mémoire retrouvée », avançait des arguments pour défendre la thèse contraire. C'est hélas un tel mouvement de balancier qui nous guette aujourd'hui, lorsque l'on confond le psychique et le factuel, lorsque l'on fétichise la parole de l'enfant ou lorsque l'on affirme la réalité d'un abus sexuel à partir d'un seul dessin d'enfant promu au rang de preuve. La clinique ne peut réussir là où le « sérum de vérité » et le « détecteur de mensonge » ont échoué.

Avec une telle prétention, elle les rejoindrait dans les poubelles de l'Histoire de la bêtise scientiste. Le clinicien doit rester étranger à toute passion militante, à toute théorie prétendument générale. Seule la singularité d'un cas, dont il ne peut d'ailleurs connaître tout le contexte, doit retenir son attention. Ses règles de conduite sont la prudence, l'humilité, le doute critique et la résistance à toutes les pressions.

Est-ce à dire qu'il faut renoncer à toute appréciation clinique? Évidemment non.

La mission de l'expert qui examine une victime présumée est centrée sur le retentissement et la crédibilité. Il recueille le récit et note le cortège symptomatique apparu après les faits dénoncés.

Mais l'existence d'un retentissement psychique ne signe pas ipso-facto la réalité des faits allégués dans les circonstances dénoncées. Il faut le rappeler à chaque fois. De même, l'expert recherche une activité délirante, des signes de mythomanie, une tendance à l'affabulation ou à la rêverie imaginative, à la conviction passionnelle, à la suggestibilité... En l'absence de tout facteur pathologique, la victime présumée est crédible sur un plan médicolégal. Cela ne signifie évidemment pas qu'elle n'a pas menti, qu'elle n'a pas été engluée dans des enjeux de loyauté ou dans des distorsions relationnelles, ce que l'expert ne peut apprécier à coup sûr dans le cadre nécessairement limité d'un examen d'expertise. Il faut le dire à chaque fois. La victime présumée peut donc être parfaitement crédible au plan médico-légal et ne pas révéler la vérité des faits. Le champ de la crédibilité médico-légale et celui de la vérité ne se superposent pas. Il faudrait être singulièrement naif pour imaginer une telle adéquation miraculeuse, qui réduirait tragiquement la complexité des conduites humaines.

La vérité des faits ne peut pas plus sortir de la bouche de l'expert que de la bouche de l'enfant. Elle se construit péniblement en un puzzle hétérogène qui assemble patiemment des données objectives et subjectives, les recoupe et les confronte sur la durée.

C'est l'affaire de la justice. Il n'est pire désastre que de prendre à la lettre une accusation proférée dans un moment de souffrance individuelle ou collective, d'y apposer le sceau de la science et d'y ajouter de terribles effets : faire apparaître désormais le sujet comme un menteur, voire comme un pervers, dans un retournement de la dénonciation. L'excès passionnel ou la conviction militante nuisent à la cause que l'on croit servir. Et puisqu'il ne faut pas hésiter parfois à énoncer des truismes : défendre la dignité de la femme ou de l'enfant, c'est promouvoir des principes de progrès et des valeurs. Mais croire de façon simpliste que toutes les souffrances qui interpellent une association d'aide aux victimes ou la justice sont l'expression ou l'illustration de ces principes, est une illusion dramatique. Ce qui s'avèrera vrai ici, sera faux là, douteux ailleurs. C'est à la justice d'en décider sereinement au cas par cas, avec l'aide d'experts qui ne débordent pas du champ de leur compétence et qui seraient bien avisés de retenir les leçons de l'Histoire

En médecine, comme dans les sciences humaines, il n'y a pas de science sans conscience de ses limites et la seule bonne conscience n'est pas la science.

\section{RÉFÉRENCES BIBLIOGRAPHIQUES}

1. Canguilhem G. le normal et le pathologique. Paris : PUF, 1966.

2. Freud S (1906). La psychanalyse et l'établissement des faits en matière judiciaire par une méthode diagnostique. Essais de psychanalyse appliquée. Paris : Gallimard, 1971.

NdIR : Notre collègue Daniel Zagury a obtenu le Prix Évelyne et Jean Kestemberg 2003 pour son article « Les serial killers sont-ils des tueurs sadiques », paru dans la Revue Française de Psychanalyse (2002, tome LXVI, n², p. 1195-1213). 